\title{
Response
}

\section{Increased Risk of Cardiovascular Disease and Mortality in Patients with Diabetes and Coexisting Depression: A Nationwide Population-Based Cohort Study (Diabetes Metab J 2021;45:379-89)}

Inha Jung, Eun-Jung Rhee, Won-Young Lee

Division of Endocrinology and Metabolism, Department of Internal Medicine, Kangbuk Samsung Hospital, Sungkyunkwan University School of Medicine, Seoul, Korea

We would like to thank Jin Hwa Kim for her valuable comments and interest in our article, entitled "Increased risk of cardiovascular disease and mortality in patients with diabetes and coexisting depression: a nationwide population-based cohort study" [1]. We are pleased that Kim recognized the value of our study, which is reflecting the effects of psychologic aspects on cardiovascular disease (CVD) among diabetic patients in Korea.

In our study using the Korean National Health Insurance Service and claims data, we found that participants with diabetes and prolonged coexisting depression have higher risk for CVD and all-cause mortality than patients who required short-term treatment for depression. The global prevalence of depression in the general population has significantly increased during the coronavirus disease 2019 (COVID-19) pandemic [2,3]. Given this situation, individualized diabetes management including psychological interventions should be provided by clinicians amid the pandemic.

We agree with Kim's opinion that further prospective studies are required to evaluate the long-term effects of interventions for depression on CVD outcomes in diabetic patients or to compare the effects on CVD between non-pharmacological interventions and pharmacological interventions for depression. To date, several trials have aimed to examine whether treatment for depression improves CVD outcomes in patients with heart disease. The Sertraline Antidepressant Heart Attack Randomized Trial (SADHART) evaluated use of an antidepressant sertraline versus placebo for depressed patients with acute myocardial infarction (MI) or unstable angina, suggesting antiplatelet and endothelium-protective properties of sertraline [4]. The Enhancing Recovery in Coronary Heart Disease (ENRICHD) trial was a randomized trial comparing cognitive behavioral therapy versus usual care following $\mathrm{MI}$ within the past 30 days [5]. However, there is not sufficient evidence regarding pharmacotherapy or psychotherapy for depression in prevention of cardiac events among Asian patients with diabetes. A large, randomized, controlled trial to address this question is required; however, patients with depression are at high risk for CVD and should not be assigned to a placebo group owing to ethical problems.

As Kim suggested, it would be helpful to consider the severity of diabetes in our study. Unfortunately, we were unable to obtain laboratory data such as glycosylated hemoglobin to assess the degree of glucose control. Future research using other

This is an Open Access article distributed under the terms of the Creative Commons Attribution Non-Commercial License (https://creativecommons.org/licenses/by-nc/4.0/) which permits unrestricted non-commercial use, distribution, and reproduction in any medium, provided the original work is properly cited.
Division of Endocrinology and Metabolism, Department of Internal Medicine, Kangbuk Samsung Hospital, Sungkyunkwan University School of Medicine, 29 Saemunan-ro,

Jongno-gu, Seoul 03181, Korea

E-mail: hongsiri@hanmail.net 
databases with more information regarding the severity of diabetes is required.

We agree with Kim's suggestions for future studies. We should take into account the potential effects of smoking and alcohol not only at study baseline, but also during the whole follow-up period. Since we could not rule out the possibility of unknown influence of comorbidities, we should exclude comorbidities such as chronic disease and cancer.

Although our observational study has several limitations, we suggest that either recurrent depression or even a single episode of depression in diabetics should be identified and treated appropriately. We would like to thank Kim again for her insightful comments on our article.

\section{CONFLICTS OF INTEREST}

No potential conflict of interest relevant to this article was reported.

\section{REFERENCES}

1. Jung I, Kwon H, Park SE, Han KD, Park YG, Kim YH, et al. Increased risk of cardiovascular disease and mortality in patients with diabetes and coexisting depression: a nationwide population-based cohort study. Diabetes Metab J 2021;45:379-89.

2. Ettman CK, Abdalla SM, Cohen GH, Sampson L, Vivier PM, Galea S. Prevalence of depression symptoms in US adults before and during the COVID-19 pandemic. JAMA Netw Open 2020;3:e2019686.

3. Salari N, Hosseinian-Far A, Jalali R, Vaisi-Raygani A, Rasoulpoor S, Mohammadi M, et al. Prevalence of stress, anxiety, depression among the general population during the COVID-19 pandemic: a systematic review and meta-analysis. Global Health 2020;16:57.

4. Serebruany VL, Glassman AH, Malinin AI, Nemeroff CB, Musselman DL, van Zyl LT, et al. Platelet/endothelial biomarkers in depressed patients treated with the selective serotonin reuptake inhibitor sertraline after acute coronary events: the Sertraline AntiDepressant Heart Attack Randomized Trial (SADHART) Platelet Substudy. Circulation 2003;108:939-44.

5. Berkman LF, Blumenthal J, Burg M, Carney RM, Catellier D, Cowan MJ, et al. Effects of treating depression and low perceived social support on clinical events after myocardial infarction: the Enhancing Recovery in Coronary Heart Disease Patients (ENRICHD) Randomized Trial. JAMA 2003;289:310616. 